\title{
Simplicity Is not Simple: How Business Architecture in One of Belgium's Biggest Companies Can Be Simple and Easy-to- use.
}

\author{
Dave De Clercq, Maxime Bernaert, Ben Roelens and Geert Poels \\ Department of Business Informatics and Operations Management \\ Faculty of Economics and Business Administration, \\ Ghent University, Tweekerkenstraat 2, B-9000 Ghent, Belgium \\ \{Dave.DeClercq, Maxime.Bernaert, Ben.Roelens, Geert.Poels\}@UGent.be
}

\begin{abstract}
As organizations are becoming more complex, Enterprise Architecture (EA) serves as an important means to align the strategy with the operations and to achieve business/IT (i.e., Information Technology) alignment. Although numerous approaches have been designed for large enterprises, little EA research was oriented towards smalland medium-sized enterprises (SMEs). However, both organizational types are fundamentally different and require a tailored approach. Therefore, CHOOSE was designed as an EA approach that is in accordance with the needs of SMEs. By performing a case study in the department of a large enterprise, this paper aims to investigate how CHOOSE can be used outside its original context. More specifically, it will be examined how the metamodel and modeling method could be adjusted to deliver an overview and valuable insights about a complex business reality. To realize this, potential solutions for the encountered issues are formulated and evaluated by the involved business stakeholders.
\end{abstract}

Keywords: Enterprise Architecture, Small- and medium-sized enterprise, CHOOSE approach, Case study

\section{Introduction}

EA is a crucial aspect to manage the complexity of an organization by using a coherent whole of principles, methods, and models that offer a holistic view on the design and realization of the enterprise's organizational structure, business processes, information systems, and infrastructure [1,2]. This holistic view of the enterprise is considered as the (end)product of an EA approach, which could be used as a communication device among various stakeholders of a company [2]. Furthermore, EA facilitates the realization of business/IT alignment as it provides insights in how to derive value by keeping information system requirements in line with the business needs $[3,4]$.

EA has become a fairly mature domain when it comes to large enterprises, but it neglected SMEs for a long time [5]. As a result, SMEs perceive most existing EA techniques 
as being complex and over-engineered [6]. Consequently, EA models are hard to understand and thus are inefficient as a communication instrument or as a support for the strategic reasoning process. This difference in perception is not surprising as SMEs and large enterprises conduct business in a fundamentally different fashion [7]. Indeed, SMEs are confronted with problems concerning lack of structure and overview [8]. In contrast, most large enterprises have elaborate organizational structures, documented in the form of an organigram. However, large enterprises cope with problems concerning complexity, which is the result of increasingly complex IT systems and of the globalization in the expanding marketspace. Moreover, knowledge is less centralized within large enterprises as they have resources for consulting aid in a full scale implementation [9]. Consequently, SMEs require a different approach than the existing EA techniques. Therefore, the CHOOSE approach was specifically designed to bring EA towards SMEs [8].

Nevertheless, the operations department of KBC Asset Management (KBC AM) showed interest in CHOOSE for a strategy-operations alignment process. As KBC AM does not qualify as an SME, CHOOSE may not be suitable in this context. Furthermore, there is no such thing as a "one size fits all" technique. Initial tests with the CHOOSE approach in the operations department yielded a complex model, which was hard to understand and thus not ideal for communication. Therefore, adaptations were needed to make the complex matter clear and useful.

The goal of this research is to investigate how CHOOSE can be used in the operations department of KBC AM. Using CHOOSE out of its intended context can yield valuable insights to make the metamodel and modeling method more robust and to increase its potential for a wider applicability in large enterprises. This includes an analysis about how the metamodel needs to be refined to be usable in the search for alignment and communication and to deliver a clear overview in a complex matter for management. Furthermore, the supporting method is discussed and compared with the existing CHOOSE modeling method.

The paper is structured as follows. In section 2, the EA approach CHOOSE is briefly explained. The research methodology (section 3) guides the execution of the case study in section 4. Section 5 presents the insights of this case study, which result in refinements to the metamodel and modeling method of the CHOOSE approach. Finally, section 6 provides the conclusions and suggestions for future research.

\section{CHOOSE Approach}

CHOOSE is an acronym for 'keep Control, by means of a Holistic Overview, based on Objectives and kept Simple, of your Enterprise'. In other words, this EA approach wants to control the complexity of an SME by offering a holistic overview of its essential organizational elements, such as the corporate strategy, the business processes, the information systems, and IT systems [10]. Furthermore, this approach takes into account the specific characteristics and attributes of SMEs by adhering to six requirements for the adoption and successful use of IT in SMEs [10]: (i) the approach should enable SMEs to 
work in a time-efficient manner on strategic issues, (ii) a person with limited IT skills should be able to apply the approach, (iii) it should be possible to apply the approach with little assistance of external experts, (iv) the approach should enable to make descriptions of the processes in the company, (v) the CEO must be involved in the approach, and (vi) the expected revenues of the approach must exceed the expected costs and risks. Consequently, the application of the CHOOSE approach will result in an EA model, which is understandable and adaptable by non-experts in an SME. Therefore, the approach was explicitly designed based on simplicity, which supports the communication between the various stakeholders [1] and is key in controlling the complexity of an organization [10].

The CHOOSE approach consists of a metamodel, a modeling method and tool support. The metamodel development was guided by requirements to enable SMEs to create simple, but comprehensive models of their enterprise [8]. The model presents a holistic overview of the organization by using elements of the business, information, and technology EA perspectives [11]. The metamodel (see figure 1) consists of four viewpoints that address a specific dimension: (i) the strategic goal dimension (i.e., yellow color), (ii) the actor dimension (i.e., red color), (iii) the operation dimension (i.e., purple color) and (iv) the object dimension (i.e., green color).

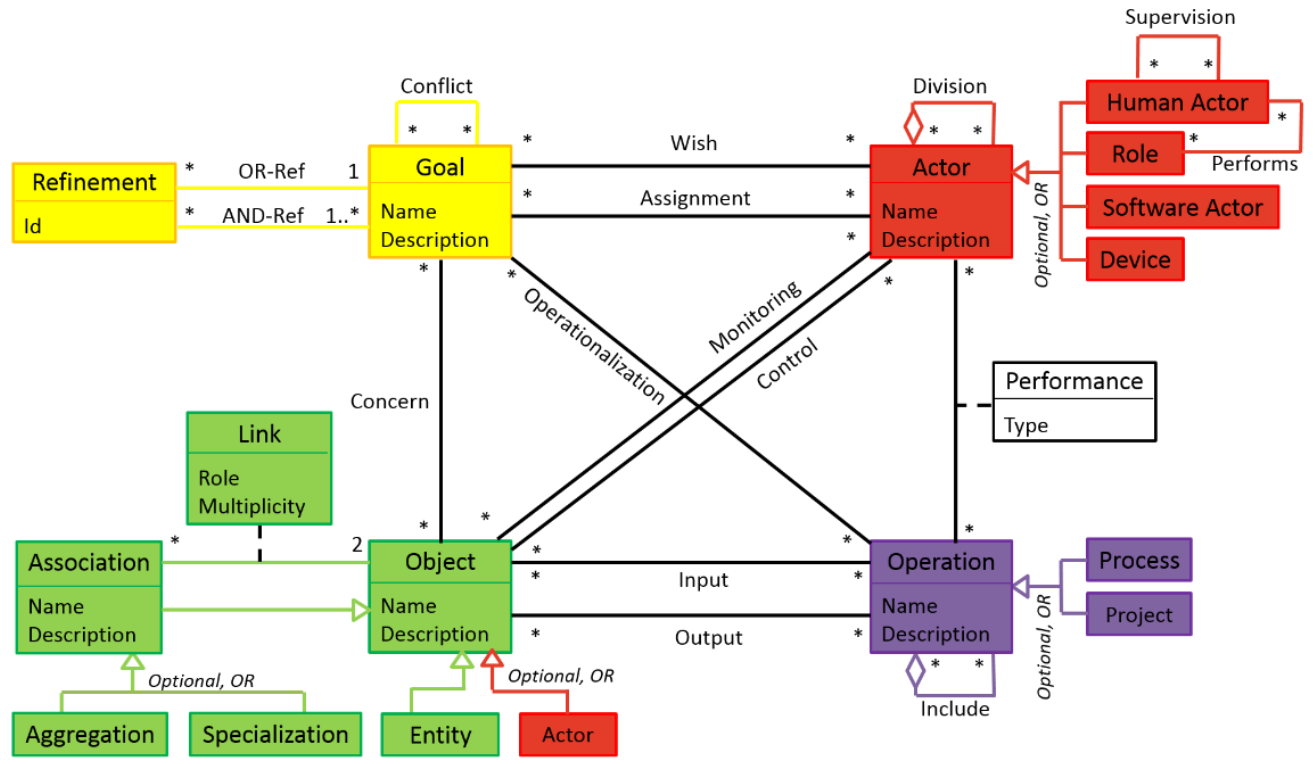

Fig 1. CHOOSE metamodel [8].

The CHOOSE modeling method consists of a six-stage roadmap, supported by a threestep interview-method and two-fold stop criteria for each dimension [12]. The roadmap guides the construction of a CHOOSE model and is built around two frameworks: (i) the Balanced ScoreCard (BSC) [13] and (ii) Porter's value chain [14]. It starts with defining high-level goals for each of the four BSC dimensions. In a second step, these goals are broke 
down to lower-level objectives by using why-how questioning [7]. Thereafter, the actor, operation and object dimensions are added to the model. The interview-method describes best practices to structure interviews with stakeholders, while the stop criteria delineate when a model is complete. Tool support is the last element of the CHOOSE approach, which has already been developed for different platforms (e.g., see [15-17]). These software tools are a crucial element in the process of bringing EA towards SMEs, as it lowers the threshold and increases the rate of adoption [12].

\section{Methodology}

This research is situated in the field of Information Systems, which is concerned with the interaction between social and technical issues. In other words, the emphasis is put on the link between human and social aspects within an organizational setting and the hardware and data aspects of IT [18]. Qualitative case study research was used as Information Systems research strategy to describe the organizational phenomena in its natural setting and to grasp the complexity of this setting. This included the use of interviews as the main method for obtaining information. Nevertheless, other data collection methods were used to increase the reliability of the data (i.e., physical artifacts, documentation and archival records) [19]. As the only way to access this information is trough social constructs (e.g., language), an interpretive perspective was adopted in this research. This perspective is often chosen in the Information Systems field to capture human interpretations concerning computer-based information systems in a business context [20].

The case study was performed by an outside observer. In this way, business people could freely express their opinions with a limited external influence on the situation [20]. The main disadvantage of this choice is trust, which can result in less access to sensitive information. However, a non-disclosure-agreement solved this trust issue in practice. Interview rounds were held with the CHOOSE architect and two other business stakeholders, who were involved in the project. Although the people in the operations department are dealing with conceptual modeling on a day-to-day basis, they were novices when it comes to EA modeling.

The first round of interviews resulted in a list of issues encountered by KBC AM concerning the metamodel. This list was prioritized by a single-criterion AHP process [21] and these issues were subsequently tackled by a review of relevant literature. This resulted in a list of possible solutions, which were evaluated according to the design principles of Paige et al. [22] to ensure consistency with the original design principles of the CHOOSE modeling language. In this respect, the relevant criteria are simplicity (i.e., no unnecessary complexity is introduced), uniqueness (i.e., no redundant or overlapping features), and consistency (i.e., language features meet design goals). This provided input for a second interview round, during which the proposed improvements were qualitatively evaluated by the involved business people. This evaluation was performed based on two criteria, which were considered as important by the stakeholders in the business context: (i) 'added value' and (ii) 'time efficiency'. 
In the first interview, the method that was used in practice was also explained. This adhoc method stands far from the existing CHOOSE modeling method, but enabled us to derive some shortcomings and promising elements. Although not explicitly evaluated, this analysis can provide a starting point to guide the further application of CHOOSE in large organizations.

\section{Case study}

\subsection{General}

KBC Bank, which was founded in 1998, is a subsidiary of KBC group NV through a series of mergers and acquisitions since 2005 [23]. By 2015, the group is the $18^{\text {th }}$ largest bank insurer in Europe with main markets in Belgium and Eastern Europe.

$\mathrm{KBC} \mathrm{AM}$ is one of three subsidiaries of $\mathrm{KBC}$ group NV, which is mainly responsible for the management of the investment portfolios, giving investment advice and other general activities [23]. The activities are geographically dispersed in Belgium, Luxemburg, Ireland, Czech Republic and Slovakia. The office in Brussels houses the general activities, such as operations, database management and architecture. This operations department does not manage portfolios itself, which is the job of the competence centres. Each centre is specialized in a certain type of product and disposes of the appropriate supporting technology. The office in Brussels employs approximately 300 people and qualifies as a large organization according to the European definition of SMEs [24]. In addition, the banking industry places some specific demands on the architecture, coming from the BASEL II norms [1].

\subsection{Project Description}

The project was conducted in the operations department of the KBC AM subsidiary to realize two main goals: (i) creating an entrepreneurial mindset and (ii) strategy-operations alignment. The first goal included motivating the personnel to be entrepreneurs and to undertake initiatives. This was considered difficult in a static operations environment as resistance to change could be expected. For the second objective, it was preferable that the undertaken initiatives are aligned with the defined corporate and operations strategy. Therefore, it was important to explain the workforce how their daily activities contribute to the fulfilment of the strategy. This objective is closely related to the main purpose of EA as proposed by Lankhorst [1]: "Translating the goals into concrete changes to the daily operations of the company is where EA comes into play". The choice for an appropriate EA approach was guided by the belief that simplicity was key in this project, in order to enable a clear communication with the workforce. Consequently, CHOOSE's focus on simplicity and easiness-to-use made it a suitable alternative [8]. 
The operations department had two distinct applications in mind for the CHOOSE approach:

- Gap analysis: the definition of a to-be model, starting from the as-is model. The tobe model in constructed by omitting existing elements and introducing new elements in the as-is model. Comparing these two versions should result in the identification of discrepancies or redundancies.

- Impact analysis: investigating the impact of changes (e.g., omitting an existing or adding a new goal) on the other elements in the EA (e.g., employees, IT systems, business processes, etc.). During the interviews, the impact analysis was focused on 'introducing a new product' because of the rapidly changing market conditions and the shortening life cycle of banking products.

\section{CHOOSE Refinements}

\section{$5.1 \quad$ General}

The initial CHOOSE modeling efforts yielded a complex model, which prevented to offer a clear overview of the enterprise. In this context, the main issue was the diagrammatic complexity (i.e., too many model elements and relations between them) [25], which reduces cognitive efficiency and the usability of the model as a tool for strategic reasoning.

To solve this problem, it was decided to use modularization (i.e., one model for each of the three departments) to decrease the number of elements displayed in a certain model. This should lead to an easier and faster understanding of the underlying content. This is particularly useful for novice users, who have more difficulties of coping with complexity than experts [26]. However, as this approach is considering separate parts of the organization, the holistic overview may get lost. This is important as it is one of the major advantages of the CHOOSE approach [8].

Furthermore, it was not necessary to focus on all CHOOSE dimensions in the model. In this respect, viewpoints provide a means to focus on certain aspects of an architectural model [25]. The choice of these aspects is dependent on the stakeholder with whom communication takes place [1]. In the alignment project, the developed viewpoint focused on the goal dimension of the CHOOSE approach to support the communication with the employees. This allowed to illustrate how individual objectives contribute to the high-level goals and key performance indicators (KPIs). Due to confidentiality restrictions, it is however not possible to show this viewpoint here.

\subsection{Metamodel}

Issues and Solutions. During the interviews, we identified eight issues regarding the CHOOSE metamodel. In table 1, these issues are ranked according to their importance, which was determined by using a single-criterion AHP procedure [21]. In the next 
paragraphs, solutions are formulated for each problem and an alternative is chosen according to the relevant design principles of Paige et al. [22] (i.e., simplicity, uniqueness, and consistency). Afterwards, these solutions are evaluated by the business stakeholders, who were involved in the case study.

Table 1. Prioritized list of issues regarding the CHOOSE metamodel.

\begin{tabular}{|l|l|l|l|}
\hline Issue & Description & Issue & Description \\
\hline 1 & Neutral for positional changes & 5 & Complex IT structures \\
\hline 2 & Department dimension & 6 & Terminology \\
\hline 3 & Gap analysis & 7 & National dimension \\
\hline 4 & Capacity measures & 8 & Process flows \\
\hline
\end{tabular}

Issue 1: Neutral for Positional Changes. The most important problem was expressed by the CHOOSE architect as: "In a large enterprise, people often change seats. The metamodel should be neutral for this, to guarantee maintainability". 'The seats' resemble functions, modeled as a Role construct that is performed by a Human Actor (see figure 1). People change functions quite often, but the content of these functions does not. Thus, the Role construct should be modeled independently from the Human Actor construct. Due to extra constraints, this was not possible when building CHOOSE models.

A potential solution is to use the Role construct, without specifying the Human Actor that performs this Role. In this way, the model content will not be affected when people are changing seats. Furthermore, the obligatory assignment of a Human Actor to each Role resulted in artificial situations. Other solutions could be suggested, but this solution was preferred based on the uniqueness principle.

Issue 2: Department Dimension. It is possible to model business units in CHOOSE, but at KBC AM they felt the need to model actors on different levels of granularity to express their organizational structure (i.e., the different departments that are part the organization) in the model.

Potential solutions are:

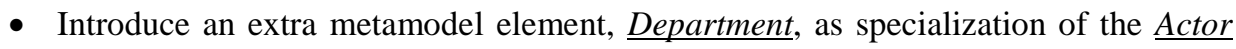
class. Consequently, the different business units, which are defined in the Division relation, make up such a department.

- Introduce a Department attribute for the aggregation relation Division. By doing this, queries should be able to show the different departments.

- Make a model for each department separately.

In this case, the second option was chosen as the explicit purpose of the Division relation is to model the organizational structure. This coincides with the uniqueness and simplicity principle. 
Issue 3: Gap Analysis. As mentioned above (cfr., sect. 4.2), a preferred application of the CHOOSE approach was the execution of a gap analysis between a to-be and an as-is model. For the goal dimension, this was realized by modeling the to-be goals as OR-refinements. All other elements, which are related to these goals, would represent the to-be model. Nevertheless, the application of a gap analysis should start from the as-is model and should include all CHOOSE dimensions.

Potential solutions are:

- Develop separate models for the as-is and to-be situation, starting from identical elements.

- Use AND-refinements and OR-refinements for all CHOOSE dimensions (as for the obstacles in the KAOS model [27]).

- Use specific symbols for the to-be elements and relations.

- Introduce gap and plateau constructs as in the mitigation extension of ArchiMate [28]. While the first construct can be used to model to-be elements, the latter denotes stable as-is elements of the EA.

Developing a to-be model comes down to introducing a new element or deleting an existing element in the as-is model, which results in a new set of relations in the model. Thus, a specific symbol for these new elements and relations could solve the experienced issue. By introducing this symbol as an attribute to the elements and relations in the CHOOSE metamodel (e.g., a Boolean variable: $\underline{\text { New }}=$ YES / NO), the to-be model can be constructed and saved starting from the as-is. Furthermore, the use of separate models is not desirable because this would lead to extensive time consumption. Moreover, option 2 and 4 would imply introducing new elements to the meta-model. Consequently, we have opted for the third solution based on the simplicity and consistency principles.

Issue 4: Capacity Measures. This issue is related to the impact analysis, during which the introduction of a new product was investigated (cfr., sect. 4.2). In this case, the impact on the available capacity should become visible. However, capacity measures are missing in the current version of the CHOOSE metamodel. As this type of changes may impact the whole organization, a decision should be taken about where to introduce these capacity attributes (e.g., employee workload constraints).

Potential solutions are:

- Adding attributes to the Role construct. A first attribute denotes the Maximal Available Capacity and the second attribute expresses the Remaining Free Capacity for a certain Role.

- To obtain a lower-level view, the attributes that are described above could be placed on the Performance link between an Actor and an Operation.

- Operations may have capacity constraints, thus adding the relevant attributes to the Input and Output relations have to be considered.

The second and third option would result in a larger number of model elements, which increases the diagrammatic complexity. Based on the simplicity and uniqueness principle, the first alternative was proposed here. 
Issue 5: Complex IT Structures. Doubts were raised about the ability of CHOOSE to model extensive and complex IT structures. Indeed, it should be able to model how the functionalities of different IT components in the organization are linked. As CHOOSE primarily focuses on the business layer, this aspect could be elaborated more.

Potential solutions are:

- Further elaborate the Object construct to enable the modeling of complex IT structures.

- Establish a link with more specialized EA approaches (e.g., ArchiMate [28]).

Based on the separation of concerns, we have opted for the second alternative. This enables to restrict the complexity of the CHOOSE approach [29]. This integration was also suggested in [8].

Issue 6: Terminology. Although the involved business stakeholders are dealing with conceptual modeling on a day-to-day basis, they struggled with the CHOOSE terminology as the project was executed. Furthermore, the CHOOSE architect needed to develop extensive knowledge to develop the model, which was an unexpected hurdle to overcome when starting modeling.

Potential solutions are:

- Only include metamodel concepts that directly relate to the business.

- Develop the CHOOSE model with the external help of an expert/consultant.

- Build an entry-level approach by using a modeling language dialect especially designed for novice users

- Explain the definitions of the elements of CHOOSE as a first step in the modeling method.

Based on the consistency and simplicity principle, the last alternative was preferred here. This has also been discussed in [8]. Although the use of an expert/consultant conflicts with the original orientation towards SMEs [10], large enterprises are expected to have more resources available for EA. Therefore, option 2 could also be a solution.

Issue 7: National Dimension. Geographical dispersion is an aspect that is typically associated with large enterprises rather than with SMEs. This aspect was not yet present in the metamodel.

Potential solutions are:

- The introduction of a Location construct that is linked with the other metamodel constructs.

- Add optional Location attribute to $\underline{\text { Actor }}$, Operations and/or Objects. For larger enterprises, Goals typically do not change over national boundaries.

Based on the simplicity principle, the second alternative was preferred. Indeed, this solution enables the modeler to decide which elements of the metamodel should have this attribute. For this specific case, the Location attribute was only relevant for $\underline{\text { Actor }}$ and Objects, as the names of the Operations differ across national boundaries. 
Issue 8: Link with Process Flows. Modeling process flows is not possible in CHOOSE. This is a deliberate choice as this aspect is adopted by process modeling languages (e.g., BPMN [30]). This clear separation of concerns preserves the simplicity of the CHOOSE approach [29]. A possible solution for this issue includes a consistent naming of actors who perform the same activity in process and CHOOSE models. By using appropriate references, this enables to integrate the information about a certain element in both models.

Evaluation. The qualitative evaluation of the proposed solutions was done by the business stakeholders and is based on the criteria they valued important: (i) added value and (ii) time efficiency. This ensured an evaluation of the proposed solutions in the relevant case study context.

Issue 1: Neutral for Positional Changes. Modeling the names of the individual employees was not considered as relevant for this case study but was nevertheless imposed during the project. As this aspect created problems, only using the Role construct could allow neutrality in the models.

Issue 2: Department Dimension. Being able to create a hierarchy of the different business units was perceived as an important aspect for the clarity of the organizational structure.

Issue 3: Gap Analysis. During the interview, the use of an extra symbol to identify the tobe model elements was preferred. In addition, the suggestion of an extra highlighting functionality aroused enthusiasm (i.e., when selecting a certain element or relation in the model, this functionality highlights every connected element). The research in [31] shows that this functionality increases the perceived usefulness of a model, which could further facilitate the gap analysis.

Issue 4: Capacity Measures. The capacity measures were considered as relevant for the $\underline{\text { Actor }} /$ Role construct, because capacity is measured in FTEs at KBC AM. Introducing more detail on the Performance relation would ask more time and add less value as this level of detail is only consulted when capacity problems arise. Although it could be useful in a production environment, the measure on the Input and Output relation of the Operation construct is not very useful in a service industry. This is mainly because these Input and Output relations are perceived as vaguer in the context of KBC and can vary according to the situation.

Issue 5: Complex IT Structures. Establishing a link with specialized EA approaches scored highest on both criteria as it allows the integration of both business and IT perspectives. During the project, it was noticed that there is considerable gap between both worlds. IT employees were primarily oriented towards the object dimension of CHOOSE and experienced problems to implement their ideas about complex IT structures. Businessminded employees focused on the other dimensions and largely neglected IT structures. Consequently, the integration of CHOOSE with a more IT-oriented EA approach was considered as an indispensable aspect. 
Issue 6: Terminology. The use of an entry-level metamodel was perceived as the best option with respect to the time-saving criterion. Besides this, it was believed that hiring a consultant would add the most value to the EA modeling. The suggestion of explaining the definitions was deemed interesting but time-consuming. Due to the higher availability of resources in contrast to SMEs, a combination of these options (e.g., the combined use of an expert and a supporting explanation of the definitions) was also seen as a feasible solution.

Issue 7: National dimension. As the location of most departments is included in their description (e.g., operations department Belgium), it was preferred to model the national dimension by means of an attribute to the relevant actors.

Issue 8: Process Flows. The functionality of developing references with related process models was conceived as a value-adding aspect. However, doubts were raised about the time that is needed to establish this integration.

\subsection{Modeling Method}

Ad-hoc Modeling Method. The project in the operations department was constructed in an ad-hoc fashion, without following the documented CHOOSE method. However, certain elements were applied similarly and other elements were added. Therefore, we first explain the alternative method that was applied during this project. Afterwards, possible additions to the existing CHOOSE method are presented. This contributes to the robustness of the CHOOSE method for a wider applicability in large organizations.

Step 1: Defining Strategy. The operations department of KBC AM was lacking a clear functional strategy to support the general strategy of KBC AM. However, conformity between both strategies is important to align all future initiatives with the overarching strategy. Therefore, the $\mathrm{COO}$ and the operations board, which consisted of the heads of the three departments (i.e., back-office, front-office, and architecture-processes-technology), defined a suitable strategy, vision, mission and KPIs (i.e., to make the goals measurable). A second aspect was adapting the employee mindset towards the entrepreneurial idea. This step lasted approximately six months to overcome the resistance to change.

Step 2: Model Building. After defining the operations strategy and its communication towards the employees, the design of the EA model began. Each department head organized workshops with the team members to define the relevant actors, objects, and strategic goals for each team. Afterwards, the goal tree was modeled and linked with the important KPIs defined in step 1. During this phase, differences in the interpretation of definitions by employees were noticed. This problem was resolved by a clarification of the keywords by the operations board. In the future, this should be done in the beginning of a project to avoid extra efforts afterwards.

Step 3: Elaboration. The EA model of the previous step resulted in a tangle of relations, which prevented clear insights. Therefore, the decision was made to concentrate on the goal dimension of CHOOSE as this aspect was the most relevant for the project. Furthermore, 
additional steps were needed to make the complex matter useful and clear, which enables to think about strategy alignment. The biggest decrease in complexity was the result of breaking the single goal tree down into more manageable parts, one for each department. This significantly decreased the number of model elements and the resulting visual complexity [25].

Step 4: Analysis. Once the model was clearly understandable, the analysis could start. Most insights were generated by reasoning about strategic issues. More specifically, by linking the goal tree with the KPIs from step 1, it was noticed in the CHOOSE model that some Goals functioned as a crossroad with a considerable higher number of paths passing through. These were named 'gateways' and underlying managerial levers were defined to obtain more detailed insights. The second part of the analysis was done by the management team, who clustered the goals according to their relevance. It was remarkable that these clusters resembled the categories of the BSC. This was interpreted as a signal that the analysis was performed in the right way. A third aspect of the analysis was performed because the enterprise architect noticed that some activities did not link to the highest-level goals. These activities were mainly situated in the Legal and Reporting business and coincide with the BASEL II norms, which are specific for the banking industry.

Step 5: Implementation. Using the identified clusters of gateways as a starting point, the department heads organized workshops with their employees. During these workshops, plans for future actions were defined with matching KPIs. The importance of the communication aspect for EA arose in this phase. Indeed, communicating the model with the employees offered the possibility of coupling daily activities with goals and according KPIs, up until the high-level goals from step 1. In this context, the model had to offer a clear overview of the EA, which was achieved by the use of viewpoints (cfr., sect. 5.1).

Step 6: Follow-up. The follow-up of this project is essential to keep the EA model a living document. This happens monthly at management level and yearly for the employees.

Additions to CHOOSE Method. In step 1, the operations board started with the definition of a strategy, vision, mission and the coinciding high-level goals. This step was performed as suggested by Kaplan and Norton, who argue that it is easier to start with the strategy/mission/vision of the organization, before formulating KPIs in the four areas of the BSC [13]. This could be a valuable addition to the roadmap of the CHOOSE Method.

In contrast with CHOOSE, building the goal tree in step 2 happened in a bottom-up manner, instead of the suggested top-down approach where the highest-level goals in each of the four BSC dimensions are further operationalized. The rationale behind this choice was to ensure a bigger involvement of the employees. A third remarkable aspect was the different interpretation of the definitions. To avoid any confusion, these definitions should be explained at the start of the project to ensure consistency in understanding. The architect also mentioned that some terminology was complicated for novice users. As already suggested by Bernaert et al. [8], a first explanatory step to clarify the main concepts could be a useful addition to the CHOOSE method. Moreover, the architect experienced the input phase as being very time consuming. To save time, it was suggested to automatically introduce 
existing models (e.g., organigrams) in the software tool. As this suggestion is related to the development of appropriate tool support, it only indirectly affects the CHOOSE modeling method.

In the analysis phase (i.e., step 4), the strategic thinking process lead to valuable insights in the form of gateways and managerial levers. Although the method was different, this process is still based on the original CHOOSE metamodel. However, finding the gateways in an automated fashion (e.g., by the formulation of appropriate queries), could increase the efficiency and usability of CHOOSE for this case study. Nevertheless, it is expected that this analysis is specific to the case study project. Consequently, adding these queries is not suggested as an adaptation to the existing method.

A final remark was that the process happened not strictly linear, but in an iterative manner. In other words, it is important to provide the flexibility to users to iteratively apply the different steps of the modeling method.

\subsection{Summary}

The most important refinements of the metamodel (see figure1) are threefold: (i) special symbols to denote elements of a to-be model, (ii) capacity measures on the Actor construct, and (iii) nationality attributes on the metamodel elements. Furthermore, the case study led to the suggestion of two extra functionalities (i.e., based on issue 3 and 8), which could be included in the existing tool support. Concerning the modeling method, starting with the development of a mission, vision, and strategy and the upfront explanation of the definitions could be valuable additions.

As CHOOSE was used out of its intended context, these suggestions should not be seen as changes to the general metamodel and modeling procedure. However, they can be useful for the further application of CHOOSE in large-scale organizations. In this context, this research is a clear illustration of the principle "there is no such thing as one size fits all".

\section{Conclusion and Future Research}

The CHOOSE approach, originally designed for SMEs, was applied in the KBC AM operations department for an operations-strategy alignment project. It can be concluded that by making minor changes to the metamodel, we would be able to model the real-life business situation of this large organization. During the execution of the ad-hoc modeling method, the discovery of gateways illustrated the ability of CHOOSE to generate insights trough a strategic thinking process. In addition to this, the approach enabled to create an overview for the management about a complex matter. From the ad-hoc modeling method, two promising additional steps the CHOOSE roadmap could be identified. However, these suggestions should be further tested before actually implementing them. 
A notion of caution needs to be introduced here. Making generalizations is very hard, especially as only one organization was the subject of study (i.e., single case study design [18]) and only few people within the organization were involved. On the other hand, our conclusions can be useful for other organizations and contexts. Therefore, certain details of the organization were made explicit to enable other researchers to interpret these results.

The result of this EA project within KBC AM is the execution of a follow-up project, in which the Target Application Architecture will be modeled by using the CHOOSE approach. In this follow-up project, it would be useful to test the proposed solutions, which will increase the generalizability of our findings.

Future research concerning these case study results includes three major topics. First, the application of CHOOSE as a means for gap and impact analysis provides interesting opportunities, even within SMEs. Therefore, these applications are currently under further investigation. Moreover, the use of viewpoints within the CHOOSE approach should be further elaborated. As the case study has shown, this enables to reduce the diagrammatic complexity of the models and facilitates the generated insights. Finally, establishing a link with ArchiMate could be a valuable addition for the CHOOSE approach. This could be particularly useful for experienced SME users, who experience the need to model IT systems in more detail [4].

\section{References}

1. Lankhorst, M.: Enterprise Architecture at Work: Modelling, Communication and Analysis. Springer, New York (2013)

2. Jonkers, H., Lankhorst, M., ter Doest, H., Arbab, F., Bosma, H.,Wieringa, R.: Enterprise Architecture: Management Tool and Blueprint for the Organisation. Information System Frontiers 8(2), 63-66 (2006)

3. Maes, R.: An Integrative Perspective on Information Management. In: Information Management: Setting the Scene, Huizing, A., De Vries, E. (eds.), pp. 11-28. Elsevier Science, Oxford (2007)

4. Sessions, R., https://msdn.microsoft.com/en-us/library/bb466232.aspx

5. Bhagwat, R.,Sharma, M.: Information System Architecture: a Framework for a Cluster of Small- and Medium-sized Enterprises (SMEs). Production Planning and Control 18(4), 283296 (2007)

6. Balabko, P.,Wegmann, A.: Systemic Classification of Concern-based Design Methods in the Context of Enterprise Architecture. Information System Frontiers 8(2), 115-131 (2006)

7. Bernaert, M.,Poels, G.: The Quest for Know-How, Know-Why, Know-What and Know-Who: Using KAOS for Enterprise Modelling. In: Salinesi, C., Pastor, O. (eds.) CAiSE '11 International Workshops. LNBIP, vol. 83, pp. 29-40. Springer, Heidelberg (2011)

8. Bernaert, M., Poels, G., Snoeck, M.,De Backer, M.: CHOOSE: Towards a Metamodel for Enterprise Architecture in Small and Medium-Sized Enterprises. Information System Frontiers in press, (2015)

9. Kroon, B., Van De Voorde, K.,Timmers, J.: High Performance Work Practices in Small Firms: a Resource-Poverty and Strategic Decision-making Perspective. Small Business Economics 41(1), 71-91 (2013) 
10. Bernaert, M., Poels, G., Snoeck, M.,De Backer, M.: Enterprise Architecture for Small and Medium-Sized Enterprises: A Starting Point for Bringing EA to SMEs, Based on Adoption Models. In: State of Art of IS Research in SMEs, Devos, J., van Landeghem, H., Deschoolmeester, D. (eds.), pp. 67-96. Springer, Heidelberg (2013)

11. Wagter, R., Van Den Berg, M., Luijpers, J.,van Steenbergen, M.: Dynamic Enterprise Architecture: How to Make It Work. John Wiley \& Sons, Hoboken, NJ (2005)

12. Callaert, M.: Business Architectuur Modellering in KMO's: Case Study Onderzoek ter Verfijning en Validatie van de CHOOSE Methode en Metamodel. UGent, Ghent, Belgium (2013)

13. Kaplan, R.,Norton, D.: The Balanced Scorecard - Measures that Drive Performance. Harvard Business Review Jan-Feb, 71-79 (1992)

14. Porter, M.: Competitive Advantage: Creating and Sustaining Superior Performance. The Free Press, New York (1985)

15. Bernaert, M., Maes, J.,Poels, G.: An Android Tablet Tool for Enterprise Architecture Modeling in Small and Medium-Sized Enterprises. In: Grabis, J., Kirikova, M., Zdravkovic, J., Stirna, J. (eds.) POEM '13. LNBIP, vol. 165, pp. 145-160. Springer, Heidelberg (2013)

16. Dumeez, J., Bernaert, M.,Poels, G.: Development of Software Tool Support for Enterprise Architecture in Small and Medium-Sized Enterprises. In: Franch, X., Soffer, P. (eds.) CAiSE '13 International Workshops. LNBIP, vol. 148, pp. 87-98. Springer, Heidelberg (2013)

17. Ingelbeen, D., Bernaert, M.,Poels, G.: Enterprise Architecture Software Tool Support for Small and Medium-Sized Enterprises: EASE. In: AMCIS '13, Chicago, Illinois (2013)

18. Berndtsson, M., Hansson, J., Olsson, B.,Lundell, B.: Thesis Projects: a Guide for Students in Computer Science and Information Systems. Springer, London (2008)

19. Yin, R.: Case Study Research: Design and Methods. Sage publications Inc., London (2009)

20. Walsham, G.: Interpretive Case Studies in IS Research: Nature and Method. European Journal of Information Systems 4(2), 74-81 (1995)

21. Saaty, T.: How to Make a Decision: The Analytic Hierarchy Process. European Journal of Operational Research 48(1), 9-26 (1990)

22. Paige, R., Ostroff, J.,Brooke, P.: Principles for modeling language design. Information and Software Technology 42(10), 665-675 (2000)

23. KBC, https://multimediafiles.kbcgroup.eu/ng/published/KBCCOM/PDF/COM_BDV_pdf _GROEP_JVS_2014_nl.pdf?

24. European Commission: The New SME Definition: User Guide and Model Declaration (2005)

25. Moody, D.: The "Physics" of Notations: Toward a Scientific Basis for Constructing Visual Notations in Software Engineering. IEEE Transactions on Software Engineering 35(6), 756779 (2009)

26. Sweller, J.: Cognitive Load Theory, Learning Difficulty, and Instructional Design. Learning and Instruction 4(4), 295-312 (1994)

27. Dardenne, A., Van Lamsweerde, A.,Fickas, S.: Goal-directed Requirements Acquisition. Science of Computer Programming 20(1-2), 3-50 (1993)

28. The Open Group: ArchiMate $囚 2.1$ Specification (2013)

29. Frank, U.: Multi-perspective Enterprise Modeling: Foundational Concepts, Prospects, and Future Research Challenges. Software \& Systems Modeling 13(3), 941-962 (2014)

30. OMG: Business Process Model and Notation (BPMN) (version 2.0) (2010)

31. Boone, S., Bernaert, M., Roelens, B., Mertens, S.,Poels, G.: Evaluating and Improving the Visualisation of CHOOSE, an Enterprise Architecture Approach for SMEs. In: Frank, U., Loucopoulos, P., Pastor, O., Petrounias, I. (eds.) POEM '14. LNBIP, vol. 197, pp. 87-102. Springer, Heidelberg (2014) 